$75-82$

\title{
Metachronous presentation of metastasis from renal cell carcinoma: evaluation and management of spinal metastasis
}

\author{
Authors Joshua C Patt, Jeffrey S Kneisl \\ Institution Department of Orthopaedic Surgery and Blumenthal Cancer Center, Carolinas Medical Center, \\ Charlotte, NC, USA
}

\section{INTRODUCTION}

Surgical treatment of metastatic cancer in the appendicular skeleton is well supported in the literature. Straightforward indications include pathologic fracture and impending pathologic fracture $[1,2]$. More controversial indications for operative treatment exist regarding resection or en-bloc removal for solitary metastases or other painful metastases [3]. Overall, surgical decision making must be tempered by the patient's overall performance status (ECOG, Eastern Cooperative Oncology Group), tumor type (with relation to expected survival and relative radiosensitivity) and the patient's perceived ability to recover from surgery [A, 4].
As controversial as surgical indications are in the appendicular skeleton, they are at times even more contentious in the axial skeleton. Issues such as established neurologic deficit as well as impending neurologic decline are compounded by uncertain criteria for stability and pathologic fracture. The purpose of this current report is to describe the unusual presentation of a symptomatic spinal metastasis in the setting of systemic disease, review the indications and treatment and then consider what was done using an "evidence-based medicine approach". 


\section{CASE REPORT}

A 64-year-old woman was seen in surgical orthopedic oncologic consultation for a new metastasis to the right distal femur. She was originally diagnosed with renal cell carcinoma 8 years prior, with metastatic disease to her mediastinal lymph nodes discovered 5 years later but had been considered in a stable disease state on systemic therapy with a tyrosine kinase inhibitor. In addition to her femoral disease she had a several year history of low back pain and occasional radiating ipsilateral right lower extremity pain. Her femur showed a lytic metastasis of the distal femoral metastasis. This was treated with curettage, local adjuvant (hydrogen peroxide and electrocautery) and poly-methyl-methacrylate (PMMA) augmentation. Her back pain was treated nonoperatively at this time. Approximately 1 year later, the patient reported new pain in the left thigh associated with activity. A new technetium bone scan identified two distinct lesions in the left femur and an additional lesion in the left ischium. Options were discussed with the patient and it was decided to proceed with surgical treatment of the femoral lesion for a symptomatic metastasis and to address the ischial lesion at the same surgical setting.

\section{Fig 1 Coronal MRI femur’07}

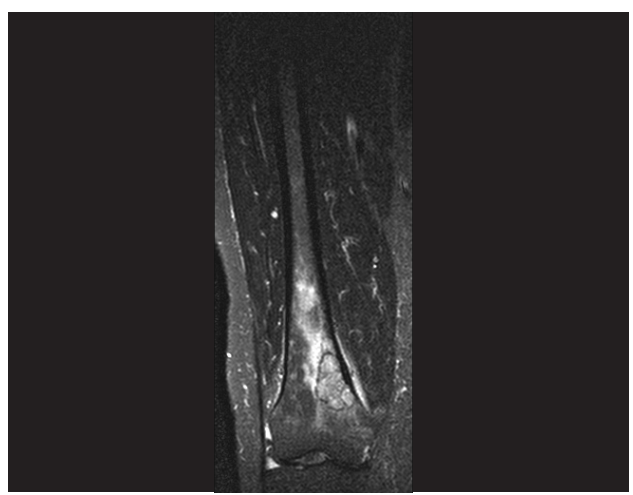

Fig 3 Sagittal MRI spine ‘07

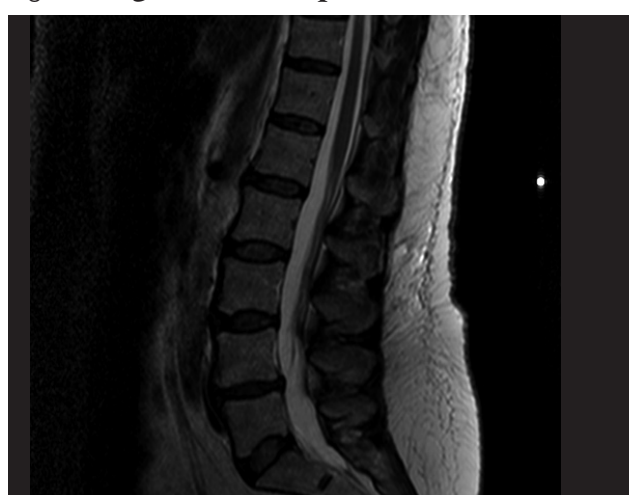

The femoral lesions were treated with simple intramedullary nailing. The ischial lesion was embolized preoperatively and then treated with curettage and local adjuvant (hydrogen peroxide [5] and electrocautery) followed by packing of the cavity with PMMA. Operative approach for the ischial lesion was a straight posterior approach as utilized for hamstring avulsion repairs. The sciatic nerve was identified and manipulation minimized. Postoperatively the patient had severe left-leg sciatic symptoms requiring escalating doses of narcotics and gabapentin therapy.

After several weeks of minimal improvement, the patient was given an L4/5 translaminar epidural steroid injection. This did provide her with moderate pain relief and allowed her to slowly wean her narcotic requirement. After a slow return of her left leg pain, a second injection was given 3 months later with similar but slightly less pain relief. This postoperative course was complicated by the patient also receiving radiation therapy to $3000 \mathrm{~Gy}$ to her ischium and left femur.

Six months following her last surgery the patient returned for an unscheduled visit with 2 weeks of crescen-

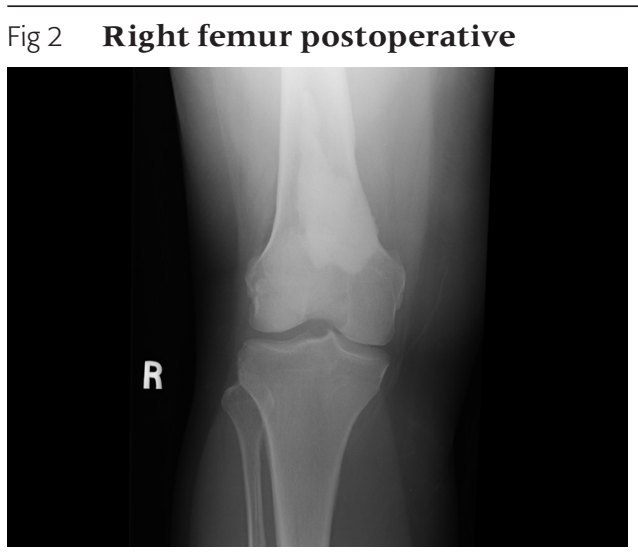

\section{Fig 4 Left ischial metastasis}

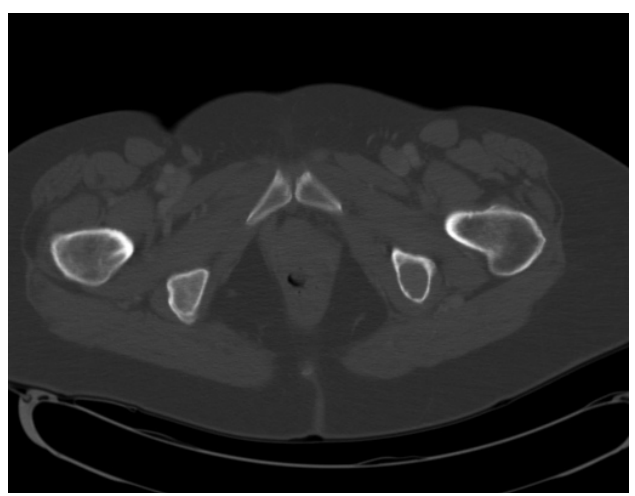


do low back pain and radiation down her bilateral posterior thighs. Noting the significant change in her pain without obvious inciting event, a new CT and MRI were ordered of her lumbar spine. These studies revealed a lytic lesion in her right L5 pedicle with expansion of the pedicle and right-sided nerve root impingement. Options were discussed with the patient including radiation therapy, embolization, surgical treatment and various combinations of the above. Based on her baseline degenerative lumbar spine problems, acute pain exacerbation, and the relatively poor radiation sensitivity of this tumor, it was decided to proceed with surgery.

\section{Surgical intervention}

Following preoperative embolization within 24 hours of planned surgery, we performed a wide posterior approach and placed pedicle screws bilaterally at L4 and S1 and unilaterally on the left side of L5. Inferior facetectomy of L4 was performed along with laminectomy of L5 to allow isolation of the L5 posterior elements and pedicle. At this point the tumor was excised in an intralesional manner. Gross total excision of the tumor was performed with resection of the entire pars, inferior articular facet, trans-

\section{Fig 5 Intraoperative left femur and ischium (patient} in prone position)

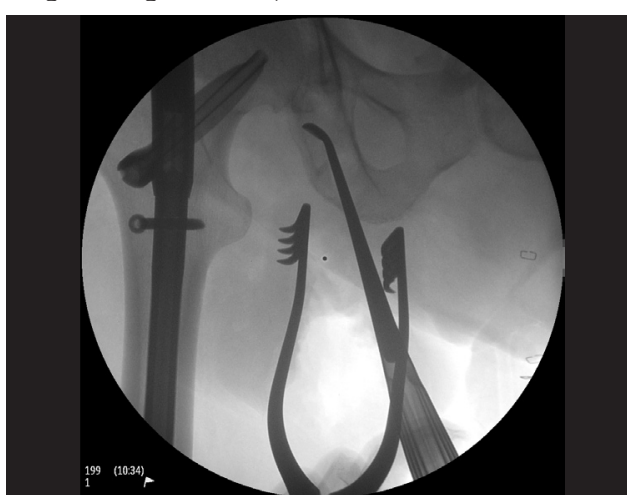

Fig 7 Para-sagittal MRI spine ‘09

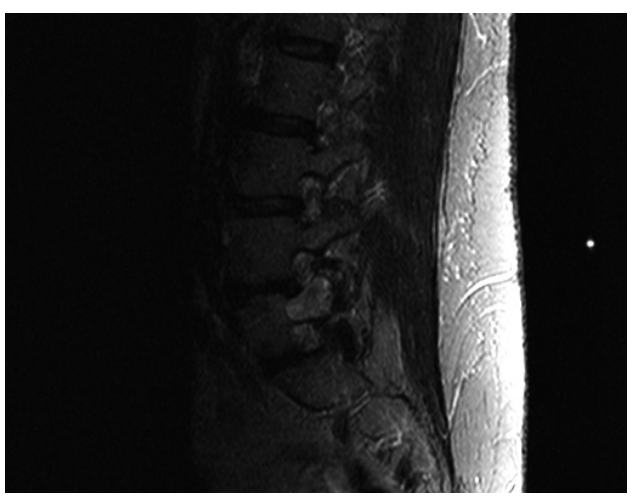

verse process and pedicle down to the vertebral body of L5. Bleeding was well controlled due to a thorough preoperative embolization and a controlled and methodical tumor resection.

After local adjuvant neoplasia treatment with peroxide, electrocautery and a high speed diamond tip burr, the L4/5 and L5/S1 disks were removed and posterior lumbar interbody fusion was carried out with transforaminal interbody allograft cages, local autograft and cancellous allograft bone and posterolateral arthrodesis completed with decortication, bonegraft placement and placement of rods and crosslink. Meticulous wound closure with nonresorbable sutures, intended to be left in place for an extended time, was carried out. The patient's postoperative course was unremarkable. She did receive postoperative radiation therapy beginning at 3 weeks postoperatively. The patient had an unremarkable postoperative course, was off of narcotic pain medicines by the 2-week follow-up visit and has had durable pain relief of her baseline back pain and her lower extremity radicular symptoms with no evidence of tumor persistence or recurrence at short-term (6-month) follow-up.

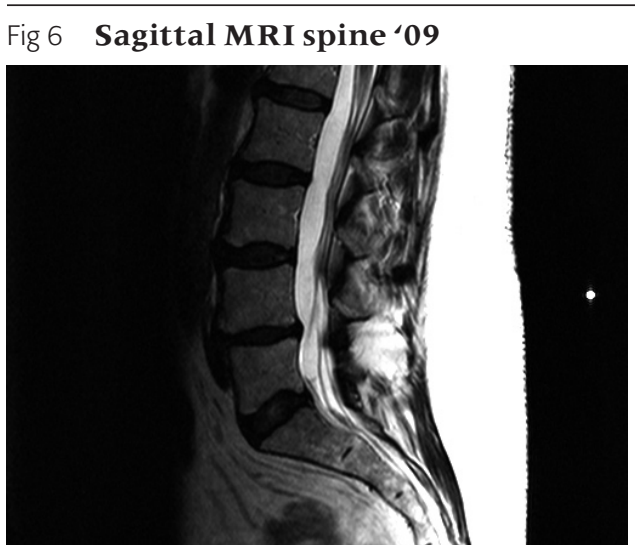

Fig 8 CT with L5 metastasis

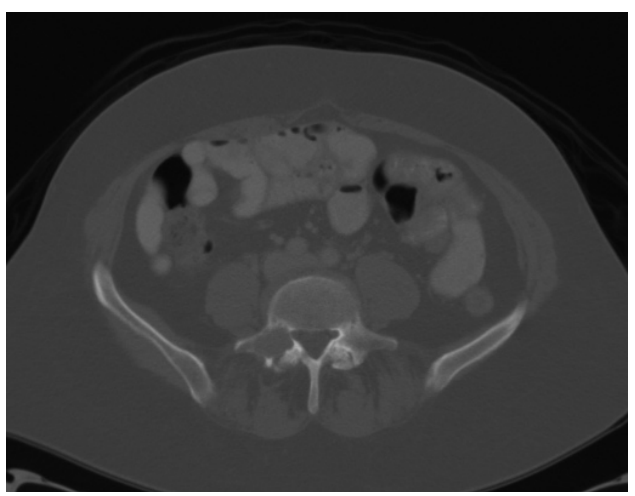




\section{Learning points}

Patients with metastatic cancer should undergo scheduled surveillance staging studies relevant to their given disease. Timing intervals and choice of studies can be selected based on established guidelines such as the National Comprehensive Cancer Network [6].

Surgical treatment of metastatic disease does not reliably provide a cure for patients in isolation. All patients should be managed by a multidisciplinary team which can include surgeons as well as medical oncologists and radiation oncologists. Metastatic disease represents a systemic disease and the only meaningful opportunity for a cure will necessitate systemic therapy which can include traditional cytotoxic agents as well as hormonal therapies or novel targeted chemotherapy agents (for instance tyrosine kinase inhibitors).

Each type of primary cancer has a unique natural history. Patients with certain tumors such as lung cancers have a typically short expected life span versus those whose tumors such as breast or renal cell which can have a much more indolent but progressive course $[7,8,9]$. Any operation considered should include a thoughtful discussion with the patient regarding the expected recovery from the planned intervention in the context of their expected longevity. For a major intervention such as spine surgery, patient life expectancy less than 3 months has been considered a contraindication for spine surgery.

The radiosensitivity of the offending tumor is a very important variable. For example, patients with myeloma or lymphoma rarely require surgical intervention due to their response to chemotherapy and radiation therapy. Breast cancer is also frequently responsive to traditional external beam radiation therapy whereas renal cell car- cinoma and melanoma are remarkably insensitive for the treatment of bulky disease [10].

In terms of clinical progression a patient with an established cancer diagnosis deserves a low threshold for ordering advanced imaging studies with the onset of new or crescendo pain. This patient had confounding baseline low back pain and then experienced nonspecific exacerbation of radicular pain following surgical intervention for her tumor disease in another region. Listening to the patient and not relying on a negative result from a bone scan prompted the treating physician to order a new MRI when the character and severity of the pain changed.

The choice of surgical intervention is frequently challenging in these patients. Attempted en-bloc resection in the appendicular skeleton has been discussed extensively and does not convincingly result in a durable cure. Consideration can be given to this type of resection in the setting of solitary metastases [B, 11]. Unfortunately the likelihood of being able to achieve an en-bloc resection with true negative margin in the spine is low and the added risk is typically not justified by the expected benefit. Patients also almost uniformly get postoperative radiation therapy and this can also adversely affect fusion rate [12]. The length of the reconstruction construct should be considered and a longer construct is typically a better option. Despite thoughtful interventions, local recurrence is more of the rule than the exception, with implications for possible future surgery in case of tumor recurrence. For example, in the present case the rods were left long caudally intentionally. The side-loading system utilized (Synthes USS, Synthes, Paoli, PA) in this case would allow the placement of iliac bolts with the current construct left intact and proximal extension also possible without extensive local re-exploration in a vascular, postirradiated bed.

\section{Fig 10 Postoperative spine AP}

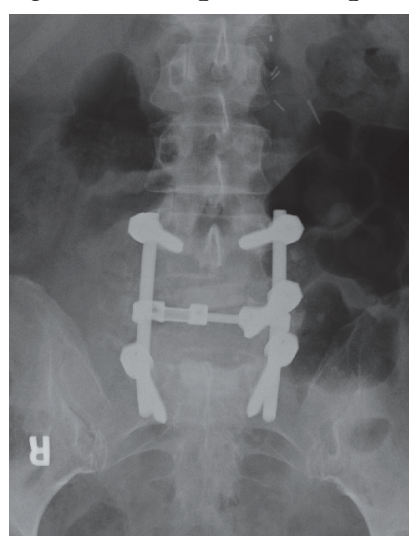




\section{CONCLUSION}

The current gold standard regarding the surgical treatment of metastatic spine disease largely relies on a study by Patchell et al, which recommended direct decompressive surgery for non-myeloid spine tumors with epidural spinal cord compression and either neurologic deficit or impending compromise [C]. Unfortunately, this article didn't address disease manifestation at the root level. Our further resources are mainly limited to the natural (treated or untreated) history of cancers from a variety of different origins, anticipated responsiveness of these tumors to radiation therapy and guidelines regarding the pre-operative evaluation and selection of appropriate surgical candidates. Unfortunately this is a complex decision making process, without concrete answers in validated reference materials. This leads to individualized treatment approaches using multidisciplinary resources, including radiation and medical oncologists.

\section{REFERENCES}

\section{Key references}

A. Tokuhashi Y, Matsuzaki H, Toriyama S, et al (1990) Scoring system for the preoperative evaluation of metastatic spine tumor prognosis. Spine; 15:1110-1113.

B. Sundaresan N, Rothman A, Manhart K, et al (2002) Surgery for solitary metastases of the spine; Rationale and results of treatment. Spine; 27:1802-1806.

C. Patchell RA, Tibbs PA, Regine WF, et al (2005) Direct decompressive surgical treatment of spinal cord compression caused by metastatic cancer: A randomized trial. Lancet; 366:643-648.

\section{Other cited references:}

1. Mirels H (1989) Metastatic disease in long bones. A proposed scoring system for diagnosing impending pathologic fractures. Clin Orthop Relat Res; 249: 256264.

2. Harrington KD (1986) Impending pathologic fractures from metastatic malignancy: evaluation and management. InstrCourse Lect; 35:357-381.

3. Bickels J, Kollender Y, Wittig JC, et al (2005) function after resection of humeral metastases: analysis of 59 consecutive patients. Clin Orthop Relat Res; 437:201208.

4. Oken MM, Creech RH, Tormey DC, et al (1982) Toxicity and response criteria of the Eastern cooperative Oncology Group. Am J Clin Oncol; 5:649-655.

5. Nicholson NC, Ramp WK, Kneisl JS, et al (1998) Hydrogen peroxide inhibits giant cell tumor and osteoblast metabolism in vitro. Clin Orthop Relat Res; 347:250260.

6. www.nccn.org

7. Althausen $\mathbf{P}$, Althausen A, Jennings LC, et al (1997) Prognostic factors and surgical treatment of osseous metastases secondary to renal cell carcinoma. Cancer; 80:1103-1109.

8. Koizumi M, Yoshimoto M, Kasumi F, et al (2003) Comparison between solitary and multiple skeletal metastatic lesions of breast cancer patients. Ann Oncol; 14:1234-1240.

9. Sugiura H, Yamada K, Sugiura T, et al (2008) Predictors of survival in patients with bone metastasis of lung cancer. Clin Orthop Relat Res; 466:729-736.

10. Frassica DA (2003) General principles of external beam radiation therapy for skeletal metastases. Clin Orthop Relat Res; 415 Suppl:S158-164.

11. Tomita K, Kawahara N, Kobayashi T, et al (2001) Surgical strategy for spinal metastases. Spine; 26:298-306.

12. Bouchard JA, Koka A, Bensusan JS, et al (1994) Effects of irradiation on posterior spinal fusions: A rabbit model. Spine; 19:1836-1841. 


\section{EVIDENCE-BASED DISCUSSION OF CASE REPORT}

\section{Author Laurence D Rhines}

Institution University of Texas, MD Anderson Cancer

Center, Department of Neurosurgery, Houston, Texas

This is a case of a 64-year-old woman with a long history of renal cell carcinoma. This was originally diagnosed approximately 9.5 years prior to her spinal surgery and, following her nephrectomy, she remained disease free for 5 years. At this time mediastinal lymph node disease was identified and controlled successfully with tyrosine kinase inhibitors. More recently her disease has become more active, with progressive osseous metastasis. A right femoral lesion was treated with curettage 8 years after diagnosis, two femoral lesions and an ischial lesion were treated with nailing and curettage 9 years after diagnosis, and 6 months later she presents with a symptomatic lesion at L5 causing crescendo back pain radiating to the thighs. This was treated with preoperative embolization followed by intralesional resection and posterior stabilization. She then received standard external beam radiotherapy 3-weeks postoperatively. Six months later, her pain is relieved and she has no evidence of tumor recurrence.

Critical review of this case centers around two key issues, prognosis and treatment options:

\section{Patient prognosis factors}

a. Histology (single greatest predictor of survival)

i. Poor survival-lung, colorectal, melanoma

ii. Long survival-breast, prostate

iii. Renal cell carcinoma (intermediate, variable natural history)

b. Extent of metastatic disease

i. Visceral metastasis

ii. Bony metastasis

c. Performance status of patient

i. Karnovsky performance score

ii. Neurologic status

Clearly the goal of treatment for metastatic spine disease is effective palliation of patient's symptoms reflective of anticipated survival time. In deciding on a plan of treatment, an appreciation of the patient's overall prognosis is critical in determining how to proceed $[1,2]$. This pa- tient has had a relatively indolent renal cancer as evidenced by 10 -year survival following diagnosis. Her disease cadence has accelerated in recent years with progressive bony metastasis, however, her overall disease burden remains limited. Her visceral disease remains controlled (lymph node disease). Her bony disease, while progressive, is limited. Finally, she has good performance status. Her predicted survival warrants intervention and said intervention must provide reasonable local tumor control.

\section{Treatment options}

1. Surgical resection

a) Intralesional resection

b) En-bloc resection

2. Radiation therapy

a) Standard external beam radiotherapy

b) Spinal stereotactic radiotherapy

3. Chemotherapy

4. Combination/sequential/pulsed therapy (surgery, radiation, chemotherapy)

Successful treatment of metastatic spine disease requires an understanding of the nature of the patient's symptoms and their etiology. The type of symptoms will often dictate the method of treatment. Metastatic epidural spinal cord compression is best managed with surgical decompression and stabilization except in the most radio/ chemo-sensitive cases (lymphoma and myeloma) [C, in key references]. The treatment of root compression is more flexible due to lack of available higher-level evidence. Local/biological tumor pain typically responds to steroids and any treatment that shrinks the tumor. Mechanical pain and pain from instability are less likely to be relieved with radiotherapy and/or chemotherapy and will typically need surgical intervention ranging from cement augmentation to surgical stabilization depending on the degree of instability.

In the present case, surgical resection and stabilization was used to treat this L 5 metastasis with excellent results at 6 months. The rationale for proceeding with surgery was the acuity of the pain, baseline degenerative disease, and the radiation resistance of renal cell carcinoma. Appropriately, embolization was performed prior to surgery to reduce intraoperative blood loss. It is unclear that there was any overt spinal instability requiring surgical 
stabilization and EBRT was used postoperatively to improve local control. En bloc resection was not utilized presumably based on the patients overall disease status and progressive bony metastasis.

Of the available treatment options, chemotherapy is the least likely to provide a timely response to the patients presenting symptoms. Moreover, having failed tyrosine kinase inhibitors, the probability of subsequent systemic therapy being efficacious is reduced. Therefore, most centers would not opt for systemic therapy alone to treat this patient's L5 disease. From the surgical standpoint, en-bloc excision has been advocated as a treatment for solitary metastasis from renal cell carcinoma [1, 2]. This patient's disease, however, is not isolated to the spine. She has nodal disease as well as increasing bony involvement over the last 2 years. Intralesional excision, as performed in this case, is clearly a reasonable choice and has led to a successful outcome in this patient at 6-month follow-up. Finally, one must consider radiation options. Renal cell carcinoma is a radio-resistant histology in the context of standard external beam radiotherapy (typically administered 30 Gy in 10 fractions). In fact, this relative radioresistance was cited by the authors as a reason for not using XRT up front, although it was used as a postoperative adjuvant. It does not appear that spinal stereotactic radiosurgery was considered, and this would have been a very reasonable alternative to surgery in this patient. Spinal stereotactic radiosurgery allows for the specific targeting of the radiation to the tumor, while sparing the adjacent sensitive structures such as the neurological elements, bowel, kidneys, vessels, etc. This allows the tumor to receive a higher dose per fraction in fewer fractions (consider 24 Gy in a single fraction) dramatically increasing the biological effectiveness of the radiation. This high-dose conformal radiation has been shown to achieve rapid and significant pain relief (> 85\% of select cases), as well as high rates of local tumor control $(85 \%-90 \%)$, with very little morbidity $[3,4,5]$. Therefore based upon these more recent publications, spinal stereotactic radiosurgery, where available, might have been another reasonable option for this patient. This case report demonstrates the complexities of trying to apply principles derived from published literature to an individual. Systematic care delivery is desirable but is put to a test under the myriad of clinical variations.

\section{DISCUSSION REFERENCES}

1. Tomita K, Kawahar N, Kobayashi T, et al (2001) Surgical strategy for spinal metastases. Spine; 298-306.

2. Tokuhashi Y, Matsuzaki H, Oda H, et al (2005) A revised scoring system for preoperative evaluation of metastatic spine tumor prognosis. Spine; 30(19): 21862191.

3. Gerszten PC, Burton SA, Ozhasoglu C, et al (2007) Radiosurgery for spinal metastases: clinical experience in 500 cases from a single institution. Spine; 32(2): 193-199.

4. Yamada Y, Bilsky MH, Lovelock DM, et al (2008) Highdose, single-fraction image-guided intensity-modulated radiotherapy for metastatic spine lesions. Int $J$ Radiation Oncology Biol Phys 71(2): 484-490.

5. Chang EL, Shiu AS, Mendel E, et al (2007) Phase I/II study of stereotactic body radiotherapy for spinal metastasis and its pattern of failure. J Neurosurg Spine; 7: $151-160$. 


\section{ADDITIONAL EBM NOTES FROM THE EDITORIAL STAFF}

A 2009 EBSS report summarized studies published in the previous 20 years that compared outcomes in patients with spinal cord compression secondary to metastatic spine tumor (of various types) who received radiation plus surgery with radiation alone [EBSS Volume 7 Issue 1, 2009]. The summary below speaks to the limitations of the literature from an "evidence" perspective and to some of the challenges related to studying the question of surgical benefit in metastatic disease.

Limited evidence from one moderate quality randomized controlled trial (CoE II) [Patchell] and three retrospective cohort studies (CoE III) [Falavigna, Ghogawala, Sorensen] suggests that survival, ability to walk, continence, and functional status may be improved and pain and complications reduced in patients experiencing metastatic spinal cord compression by adding surgical intervention to radiation therapy. A higher percentage of patients receiving combined surgery and radiation versus radiotherapy alone tended to be able to walk, and to walk longer, with statistical significance being reached in two of the three studies that examined this outcome. These potential benefits should be weighed against the costs, rigors of recovery from major surgery in patients whose health is already compromised and life expectancy. In cohort studies, treatment choice based on patient presentation may bias (confounding by indication) results comparing treatments. A methodologically rigorous multicenter study may help confirm whether surgery followed by radiotherapy will improve outcomes for metastatic spinal cord compression patients.

While consideration of evidence is important, as seen in this case, attention to individual patient presentation and circumstance must inform the ultimate course of action.

\section{STUDIES INCLUDED IN EBSS REPORT}

Patchell RA, Tibbs PA, Regine WF, et al (2005)

Direct decompressive surgical resection in the treatment of spinal cord compression caused by metastatic cancer: a randomised trial. Lancet; 366(9486): 643-648.

Falavigna A, Righesso Neto O, Polesso MA, et al (2007) Metastatic tumor of thoracic and lumbar spine: prospective study comparing the surgery and radiotherapy vs external immobilization with radiotherapy. Arq Neuropsiquiatr; 65(3B): 889-895.

Ghogawala Z, Mansfield FL, Borges LF, et al (2001)

Spinal radiation before surgical decompression adversely affects outcomes of surgery for symptomatic metastatic spinal cord compression. Spine; 26(7): 818-824.

Sorensen S, Borgesen SE, Rohde K, et al (1990)

Metastatic epidural spinal cord compression. Results of treatment and survival. Cancer; 65(7):1502-1508. 